06

\title{
Механизмы поглощения терагерцового и инфракрасного излучения в пленках PZT
}

\author{
(C) Г.А. Командин ${ }^{1}$, О.Е. Породинков ${ }^{1, \uparrow, ~ И . Е . ~ С п е к т о р ~}{ }^{1}$, А.А. Волков ${ }^{1}$, К.А. Воротилов ${ }^{2}$, \\ Д.С. Серегин ${ }^{2}$, А.С. Сигов ${ }^{2}$ \\ ${ }^{1}$ Институт общей фризики им. А.М. Прохорова РАН, \\ Москва, Россия \\ ${ }^{2}$ Московский технологический университет (МИРЭА), \\ Москва, Россия \\ ฯ E-mail: oleg.porodinkov@yandex.ru
}

(Поступила в Редакцию 11 декабря 2017 г.)

Измерены и проанализированы THz-IR-спектры диэлектрического отклика плотных и пористых пленок $\mathrm{Pb}(\mathrm{Zr}, \mathrm{Ti}) \mathrm{O}_{3}$, осажденных на подложки из монокристаллических оксидов магния и алюминия, а также на кремниевые подложки с платиновым контактным слоем. Рассмотрены основные механизмы электродипольного поглощения, формирующие полосы в экспериментальных спектрах отражения и пропускания. Обсуждается зависимость параметров полос поглощения в пленках от типа подложки.

Работа в МИРЭА выполнена при финансовой поддержке Минобрнауки России в рамках проектной части государственного задания в сфере научной деятельности, проект № 11.2259.2017/4.6; работа в ИОФ РАН выполнена при финансовой поддержке Российского фонда фундаментальных исследований, проект № 15-02-02335a.

DOI: 10.21883/FTT.2018.06.46003.342

\section{1. Введение}

Твердые растворы структурного семейства перовскита $\mathrm{Pb}\left(\mathrm{Zr}_{x} \mathrm{Ti}_{1-x}\right) \mathrm{O}_{3}$ (PZT) стали объектом детального изучения сразу после их получения в 50-х годах прошлого века. Эти материалы в виде керамик и кристаллов обладают высокими значениями остаточной поляризации, достигающими $20-90 \mu \mathrm{C} / \mathrm{cm}^{2}$. Низкочастотная диэлектрическая проницаемость, составляющая не менее $10^{3}$, слабо зависит от частоты вплоть до $\mathrm{GHz}$ диапазона [1]. Согласно фазовой диаграмме, твердые растворы PZT обладают тетрагональной структурой в области больших концентраций титана и ромбоэдрической в случае больших концентраций циркония (крайние точки - классический сегнетоэлектрик $\mathrm{PbTiO}_{3}$ и антисегнетоэлектрик $\mathrm{PbZrO}_{3}$ ) [2]. Граница этих фаз соответствует примерно равным концентрациям $\mathrm{Ti}^{4+}$ и $\mathrm{Zr}^{4+}$ и практически не зависит от температуры примерно до $350^{\circ} \mathrm{C}$. В области морфотропной фазовой границы существует также несколько промежуточных фаз $[3,4]$.

Высокое значение диэлектрической проницаемости и ее относительная температурная стабильность для составов вблизи морфотропной границы определили широкое практическое использование твердых растворов PZT. Особое место занимают исследования пленочных образцов различной толщины и морфологии. Пленки PZT в составе многослойных гетероструктур нашли применение в элементах сегнетоэлектрической памяти FeRAM [5,6], пьезоэлектрических микроэлектромеханических системах MEMS [7,8], пьезоэлектрических транзисторах [9], пироприемниках [10], энергосберегающих устройствах [11], устройствах на основе эффекта магнитострикции [12-14].

Динамика кристаллической решетки тонких пленок твердых растворов PZT всесторонне исследовалась методами неупругого рассеяния нейтронов [15-17], комбинационного рассеяния света $[18,19]$, дифракции рентгеновского излучения [20,21], а также с начала 90-х годов - методами инфракрасной и субмиллиметровой спектроскопии [22-25].

Пленки сложных оксидов получают методами химического осаждения металлорганических соединений из газовой фазы (metalorganic chemical vapour depositions, MOCVD) [26,27], импульсного лазерного осаждения (pulsed laser deposition, PLD) [28], высокочастотного магнетронного распыления [29-31], гидротермальным методом [32], методами химического осаждения из растворов (chemical solution deposition, CSD), включая зольгель метод [33,34], а также методами молекулярнолучевой эпитаксии (MBE) [35].

Методы химического осаждения из растворов нашли широкое применение для формирования тонких пленок PZT, в том числе в полупроводниковом производстве FeRAM, MEMS и различных датчиков [33]. Данные пленки имеют относительно невысокую температуру кристаллизации перовскитной фазы $\left(\sim 600-650^{\circ} \mathrm{C}\right)$, высокие значения остаточной поляризации и хорошую адгезию к оксиду магния, сапфиру и платине, как стандартному проводящему контактному слою в микроэлектронике. Золь-гель метод позволяет синтезировать пленки различной толщины и пористости.

Терагерцовая и инфракрасная спектроскопии - два близких по существу экспериментальных метода изу- 
чения электродипольных возбуждений в конденсированных средах. Для них едины правила отбора, а регистрируемыми данными являются спектры пропускания и отражения. Полученные этими методами экспериментальные результаты допускают возможность анализировать их одновременно в виде единого набора данных. При расширении рабочего диапазона в более низкочастотную (радиочастотную) область с применением импедансных методов измерений [36-38] появляется дополнительная возможность построения общих панорам диэлектрического отклика путем объединения импедансных, $\mathrm{THz}$ и IR-данных.

Колебательный спектр системы твердых растворов PZT подробно изучен методом инфракрасной спектроскопии. Анализ полученных спектров с наибольшей полнотой проведен для керамических и кристаллических объемных образцов [23]. Для тонкопленочных образцов основной акцент сделан на изучении температурной эволюции центральной (CM) и мягкой (SM) мод [39], которые формируют примерно половину диэлектрической проницаемости на низких частотах (ниже $1 \mathrm{GHz}$ ) [1] и определяют ее температурную зависимость. Было установлено, что в пленках в области средних концентраций $\mathrm{Zr}-\mathrm{Ti}$ фазовый переход в сегнетоэлектрическое состояние размыт по температуре. Максимум диэлектрической проницаемости для твердых растворов смещен на низкие температуры по отношению к температуре сегнетоэлектрического перехода в пленке $\mathrm{PbTiO}_{3}$ [24].

Диэлектрический отклик пленки в составе гетероструктуры (пленка на подложке) в целом чувствителен к напряжениям и структурным искажениям, возникающим вследствие рассогласования параметров кристаллических ячеек подложки и пленки. Методы терагерцовой и инфракрасной спектроскопии позволят наблюдать возникающие при этом спектральные эффекты. Цель нашего исследования - проследить изменения электродинамических параметров пленок PZT при осаждении на различные подложки и имеющих разные морфологические особенности.

\section{2. Экспериментальные и расчетные методики}

Экспериментальные спектры пропускания и отражения исследуемых образцов исследовались на двух спектрометрах. В диапазоне волновых чисел от 30 до $4000 \mathrm{~cm}^{-1}$ спектры получены на инфракрасном Фурьеспектрометре Bruker IFS113v. Образцы представляли собой гетероструктуры - плоскопараллельные пластины оксидов магния и алюминия с осажденными на них пленками PZT. Были также приготовлены образцы с подслоем платины между пленкой и подложкой. Спектры отражения в стандартной конфигурации спектрометра измерены при угле падения $\approx 6$ градусов. Измерение спектров отражения в $s$ - и $p$-поляризованном излучении выполнено с использованием дополнительной системы плоских зеркал. Для получения линейно поляризованного излучения применен поляризатор на основе металлизированной полиэтиленовой пленки толщиной $60 \mu \mathrm{m}$, на которой алмазным резцом сделаны штрихи треугольного профиля (1200 lines/mm). Спектры пропускания измерены при нормальном падении излучения.

На низкочастотном краю диапазона, в области больших экспериментальных погрешностей стандартных инфракрасных спектрометров, измерения проведены на лабораторном субмиллиметровом ЛОВ-спектрометре (ЛОВ - лампа обратной волны) [40]. Применение для генерации излучения частотно-перестраиваемых ЛОВ обеспечило получение точных экспериментальных данных вплоть до $5 \mathrm{~cm}^{-1}$. Все измерения как спектров пропускания, так и спектров отражения выполнены на этом спектрометре при нормальном падении излучения на образец.

Методика расчета электродинамических параметров пленки в составе гетероструктуры пленка/подложка основана на применении формул Френеля для пропускания и отражения многослойной среды с учетом дисперсии комплексных показателей преломления для каждого слоя. Дисперсия для каждого слоя рассчитана с использованием модели классического гармонического осциллятора:

$$
\varepsilon^{*}(v)=\varepsilon_{\infty}+\sum_{j=1}^{n} \frac{\Delta \varepsilon_{j} v_{j}^{2}}{v_{j}^{2}-v^{2}+i v \gamma_{j}} .
$$

Для каждой резонансной линии дисперсионные параметры $\left(v_{j}-\right.$ частота, $\Delta \varepsilon_{j}-$ диэлектрический вклад и $\gamma_{j}$ - затухание) подобраны путем минимизации среднеквадратичного отклонения расчетного спектра от экспериментальных данных.

На первом этапе работы определялись дисперсионные параметры подложек, изготовленных из $\mathrm{MgO}$ и $\alpha-\mathrm{Al}_{2} \mathrm{O}_{3}$. В расчетах модельных спектров учитывался вклад многофононных процессов поглощения, формирующих асимметричные полосы в $\mathrm{THz}$ участке спектра в монокристаллах оксида магния [41] и сапфира [42]. В работе [43] было показано, что относительно малый вклад разностных процессов в поглощение в подложке не может быть проигнорирован при расчетах диэлектрического отклика пленки.

Спектр отражения слоя платины в области THz-FIR (FIR - дальний инфракрасный диапазон) рассчитан в приближении Хагена-Рубенса

$$
R(v)=1-2 \sqrt{\frac{2 \varepsilon_{V} v}{\sigma(v)}},
$$

где зависящая от частоты комплексная проводимость определена по модели Друде

$$
\sigma^{*}(v)=\frac{\sigma_{D C}}{1-i \nu \tau} .
$$

Для расчета использованы стандартные для платины параметры: статическая проводимость $\sigma_{D C} \sim$ $\sim 10^{6} \mathrm{Ohm}^{-1} \cdot \mathrm{cm}^{-1}$ и затухание $\gamma=(2 \pi c \tau)^{-1} \sim 10^{3} \mathrm{~cm}^{-1}$, 


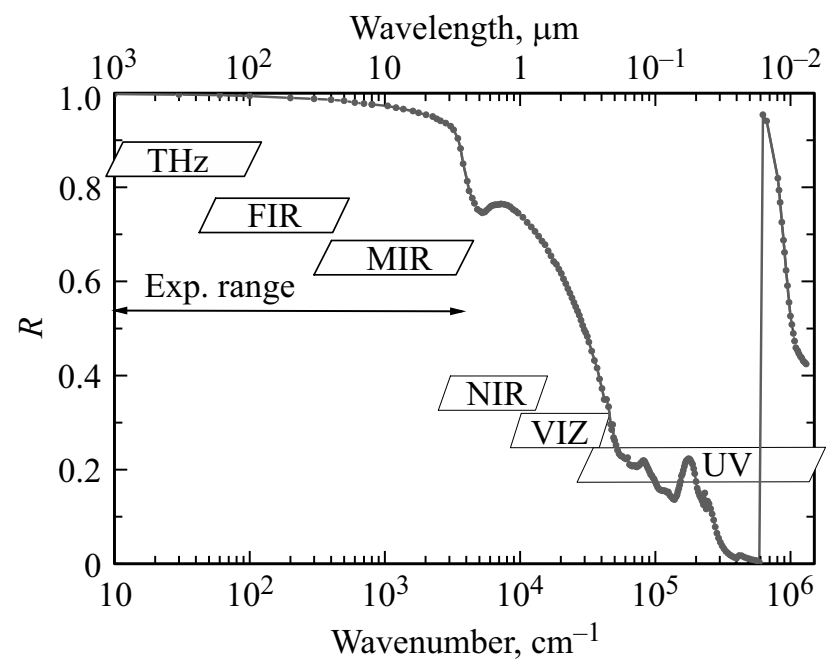

Рис. 1. Спектр отражения платины, рассчитанный по данным работы [44]. Низкочастотная часть от $800 \mathrm{~cm}^{-1}$ рассчитана по соотношению Хагена-Рубенса.

где $\tau-$ среднее время между соударениями, $c-$ электродинамическая константа, равная скорости света в вакууме. В высокочастотной области спектр отражения рассчитан по данным $n$ и $k$ из работы [44]. На рис. 1 показан полный спектр отражения слоя платины.

Для наших экспериментов пленки PZT были нанесены на вышеперечисленные подложки золь-гель методом. Пленкообразующий раствор представлял собой смесь обезвоженного ацетата свинца, тетраизопропоксида титана и моносольвата изопропилата циркония, подобранных в концентрациях, необходимых для получения пленок состава $\mathrm{Pb}\left(\mathrm{Zr}_{0.52} \mathrm{Ti}_{0.48}\right) \mathrm{O}_{3}$. Именно этот состав имеет максимальную статическую диэлектрическую проницаемость в системе твердых растворов $\mathrm{PbZr}_{1-x} \mathrm{Ti}_{x} \mathrm{O}_{3}$ [1]. Для получения толстых пористых пленок в прекурсор дополнительно вводился пороген поливинилпиролидон, который впоследствии термически деструктировался.

\section{3. Экспериментальные результаты и обсуждение}

С точки зрения спектроскопии тонких пленок с мощными электродипольными возбуждениями в $\mathrm{THz}$ области предпочтительно использовать в качестве материала подложки оксид магния. Оксид магния - кубический ионный кристалл, не имеющий анизотропии оптических и диэлектрических параметров в $\mathrm{THz}$ диапазоне (рис. 2). Двукратно вырожденный ТО-фонон, активный в инфракрасных спектрах, имеет максимум поглощения на частоте $400 \mathrm{~cm}^{-1}$ и затухание (ширину резонанса на полувысоте) $\gamma \sim 6.4 \mathrm{~cm}^{-1}$. Многофононные разностные процессы формируют сравнительно неширокую полосу в области $100-250 \mathrm{~cm}^{-1}$. На низких частотах потери определяются фононным вкладом. Дисперсия проводимости представлена низкочастотным крылом контура ТО фоно- на и определена, преимущественно, величиной константы затухания, явно зависящей от температуры [41].

В отличие от монокристалла $\mathrm{MgO}$ оксид алюминия имеет тригональную решетку с пространственной группой $R \overline{3} c$. В IR-спектре у низкочастотного фонона на частоте $385 \mathrm{~cm}^{-1}$ диэлектрический вклад $\Delta \varepsilon_{\mathrm{Al}_{2} \mathrm{O}_{3}} \sim 0.38$ $\left(\Delta \varepsilon_{\mathrm{MgO}} \sim 6.4\right)$ более, чем в 10 раз меньше, чем в случае оксида магния. В силу низкой симметрии дисперсия фононных ветвей в зоне Бриллюэна такова, что разностные многофононные процессы поглощения распределены в более широком частотном диапазоне (рис. 3). При комнатной температуре во всем $\mathrm{THz}$ диапазоне разностное поглощение увеличивает потери не менее чем на порядок величины по отношению к однофо-

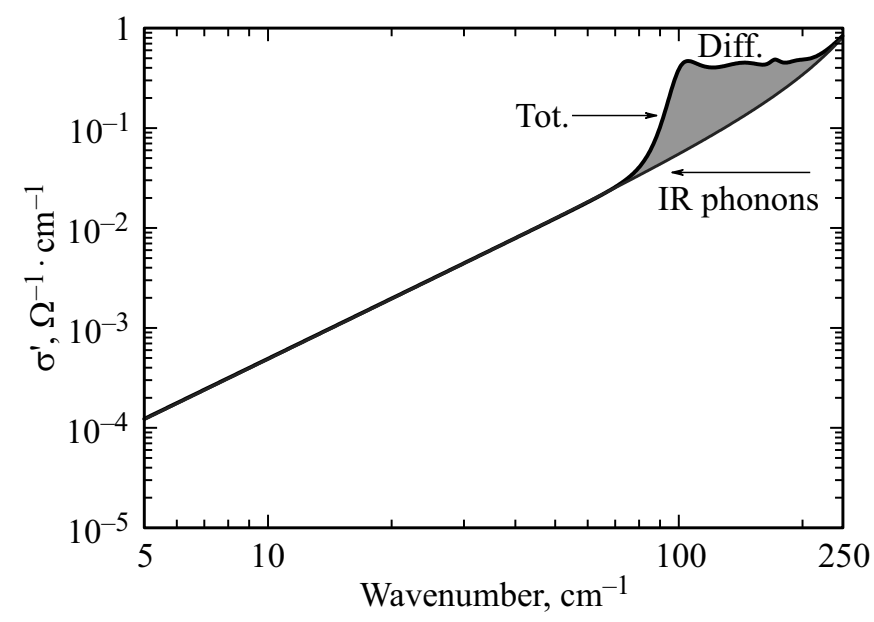

Рис. 2. Спектры динамической проводимости монокристалла

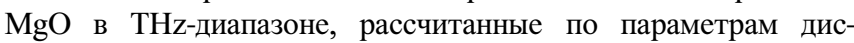
персионного моделирования. Серой заливкой выделен вклад многофононных разностных процессов.

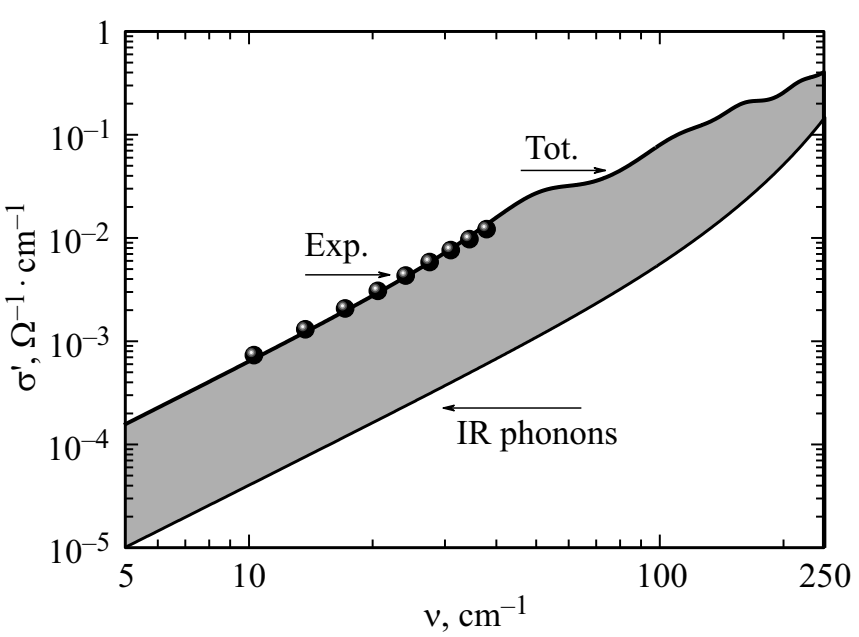

Рис. 3. Спектры динамической проводимости $\sigma^{\prime}(v)$ сапфира $\alpha-\mathrm{Al}_{2} \mathrm{O}_{3}$ в поляризации обыкновенного луча. Символы значения проводимости, определенные из интерференции в спектре пропускания по формулам Френеля. Линии - расчет спектра $\sigma^{\prime}(v)$ по параметрам дисперсионного моделирования: IR-phonons - расчет с учетом только вклада IR-активных фононов, Tot. — вклад, учитывающий все процессы поглощения. 
нонному вкладу. На рис. 2 и 3 спектры динамической проводимости оксида магния и сапфира представлены в одном масштабе. Из сравнения рисунков следует, что собственные фононные потери в сапфире на порядок меньше, чем в $\mathrm{MgO}$. Это определено большим диэлектрическим вкладом осциллятора в $\mathrm{MgO}$. Разностные процессы, увеличивая суммарные потери на порядок, сводят на нет ожидаемые преимущества сапфира.

При расчете двухслойной среды дисперсионные параметры подложек, определенные на первом этапе расчетов, фиксировались, и подбор дисперсионных параметров проводился только для слоя пленки. После нанесения, высушивания $\left(150^{\circ} \mathrm{C}\right)$ и пиролиза $\left(400^{\circ} \mathrm{C}\right)$ полученная пленка PZT является аморфной. Величина ее диэлектрической проницаемости сформирована, в основном, низкочастотными полосами в диапазоне до $100 \mathrm{~cm}^{-1}$. Суммарный диэлектрический вклад всех IR-активных полос не превышает 30, что на порядок меньше, чем у пленки в перовскитной фазе. Количественный анализ аморфных слоев возможен только для модельных пленок на диэлектрических подложках. На рис. 4 в качестве иллюстрации приведены спектры отражения подложки сапфира и подложки сапфира с нанесенной на нее аморфной пленкой PZT. Овалом на рисунке выделены высокочастотные пики, характерные для аморфных пленок.

Для аморфной пленки, осажденной на слой платины, можно сделать только качественные оценки особенностей диэлектрического отклика. На рис. 5 показаны измеренные спектры отражения-пропускания структуры пленка PZT/Pt c пленкой толщиной $\sim 100 \mathrm{~nm}$. Низкочастотная часть спектра не поддается количественному анализу. На частотах выше $1000 \mathrm{~cm}^{-1}$ узкие резонансы соответствуют тем, что зарегистрированы в PZT пленке на сапфире. Полоса на $600 \mathrm{~cm}^{-1}$ проявляется только для p-поляризации и соответствует области поглощения на

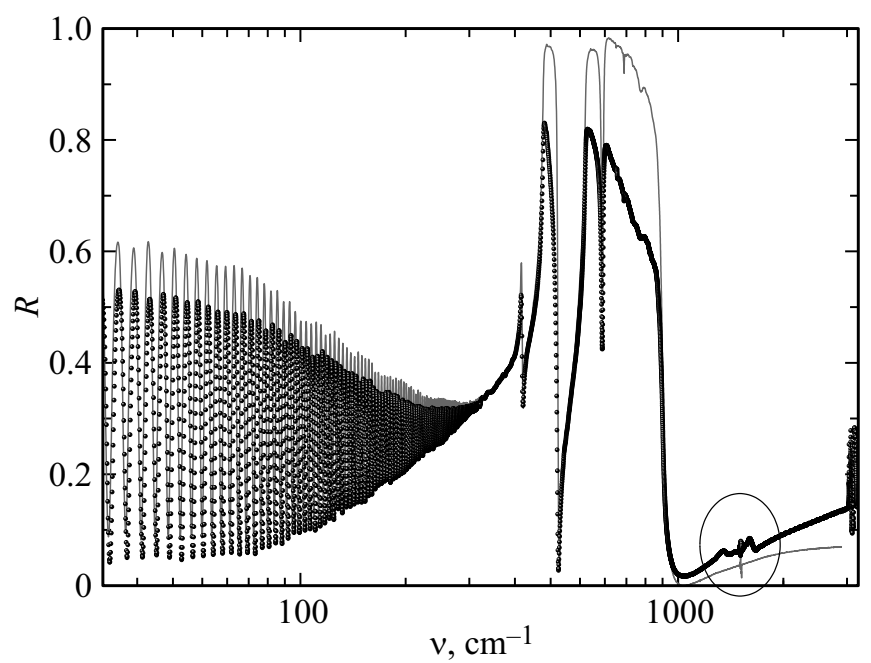

Pис. 4. Спектры отражения аморфной пленки PZT на сапфировой подложке. Символы - экспериментальный спектр структуры пленка/подложка, сплошная линия - экспериментальный спектр подложки.

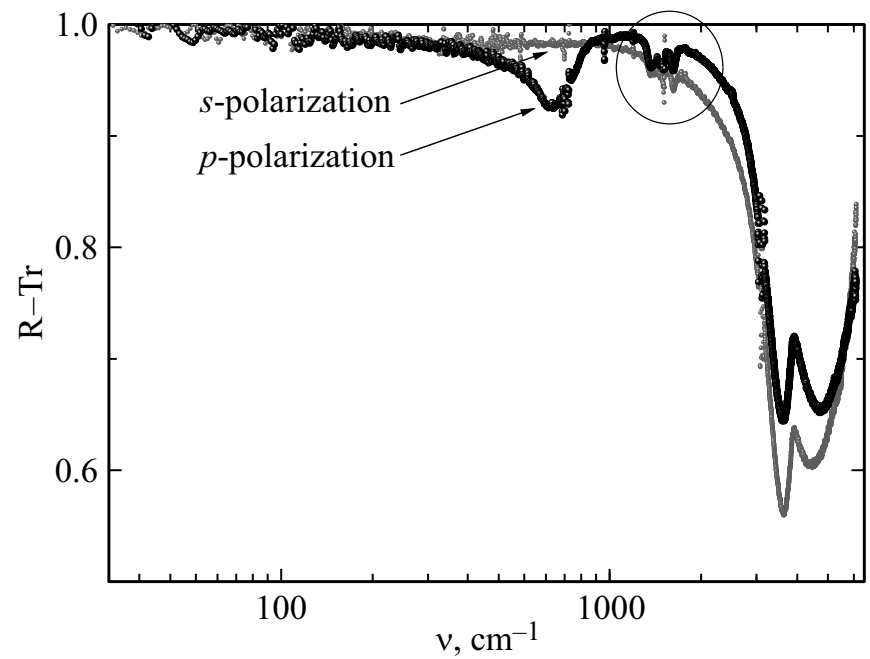

Pис. 5. Спектры отражения аморфной пленки РZT на платиновом подслое. Угол падения излучения 30 градусов.

поверхностных поляритонах на границе раздела диэлектрик/металл [45].

После кристаллизации PZT пленки при температурах выше $550^{\circ} \mathrm{C}$ [46] THz-IR спектры структур PZT/подложка трансформировались (рис. 6). Высокочастотные линии, наблюдаемые в случае аморфных пленок, полностью исчезли. В THz-IR-области проявились полосы поглощения, характерные для перовскитной фазы твердого раствора PZT.

Важным фактором, влияющим на характеристики полос поглощения, является наличие гетерофазных напряжений на границе раздела пленка/подложка из-за различных параметров кристаллической решетки подложки и осажденной на нее пленки [47]. Величина этого влияния явно зависит от материала и толщины пленки. В случае твердых растворов (Ba,Sr) $\mathrm{TiO}_{3}$ этот эффект явно проявляется в $\mathrm{THz}-\mathrm{IR}-$ спектрах пленок толщиной менее $1000 \mathrm{~nm}$ [48]. В пленках твердых растворов $(\mathrm{Bi}, \mathrm{Nd}) \mathrm{FeO}_{3}$ наличие остаточных напряжений и изменение структурных параметров было установлено и рентгеноструктурными исследованиями, на основе которых сделан вывод о релаксации параметров ячейки пленки к параметрам объемного материала. Для пленок толщиной больше $500 \mathrm{~nm}$ различия в структурных параметрах пленки и объемного материала меньше экспериментальных погрешностей [49].

Минимальное рассогласование параметров решетки у пленок PZT с платиной - 2.9\% [50]. В случае пленки PZT на подложке оксида магния эта величина составляет $4.3 \%$. Для $\alpha-\mathrm{Al}_{2} \mathrm{O}_{3}$ расхождения достигают $15.2 \%$. Возникающие в пленке напряжения приводят в определенных составах к изменению параметров структурных фазовых переходов. Типичным примером является пленка титаната стронция, осажденная на подложку из скандата диспрозия [51]. В работах [51-53] показано, что напряжения в пленке приводят к смещению температур 
структурных фазовых переходов сегнетоэлектрического и антиферродисторсионного типов.

В работе [24] установлено, что температуры структурного фазового перехода из параэлектрической в сегнетоэлектрическую полярную фазу в пленках $\mathrm{PT}\left(\mathrm{PbTiO}_{3}\right)$ толщиной $\sim 1000 \mathrm{~nm}$ на сапфировых подложках в пределах точности совпадают для объемных образцов и пленок. Показано, что в случае твердого раствора PZT переход в сегнетоэлектрическую фазу размыт по температуре. В данной работе рассмотрена температурная эволюция только мягкой моды. Сложный спектр электродипольных возбуждений сапфировой подложки делает анализ всех мод в спектре пленки не простой задачей. Кроме того, высокочастотные IR-активные фононы дают незначительный вклад в суммарную диэлектрическую проницаемость, температурная зависимость которой всецело определена эволюцией параметров мяг-
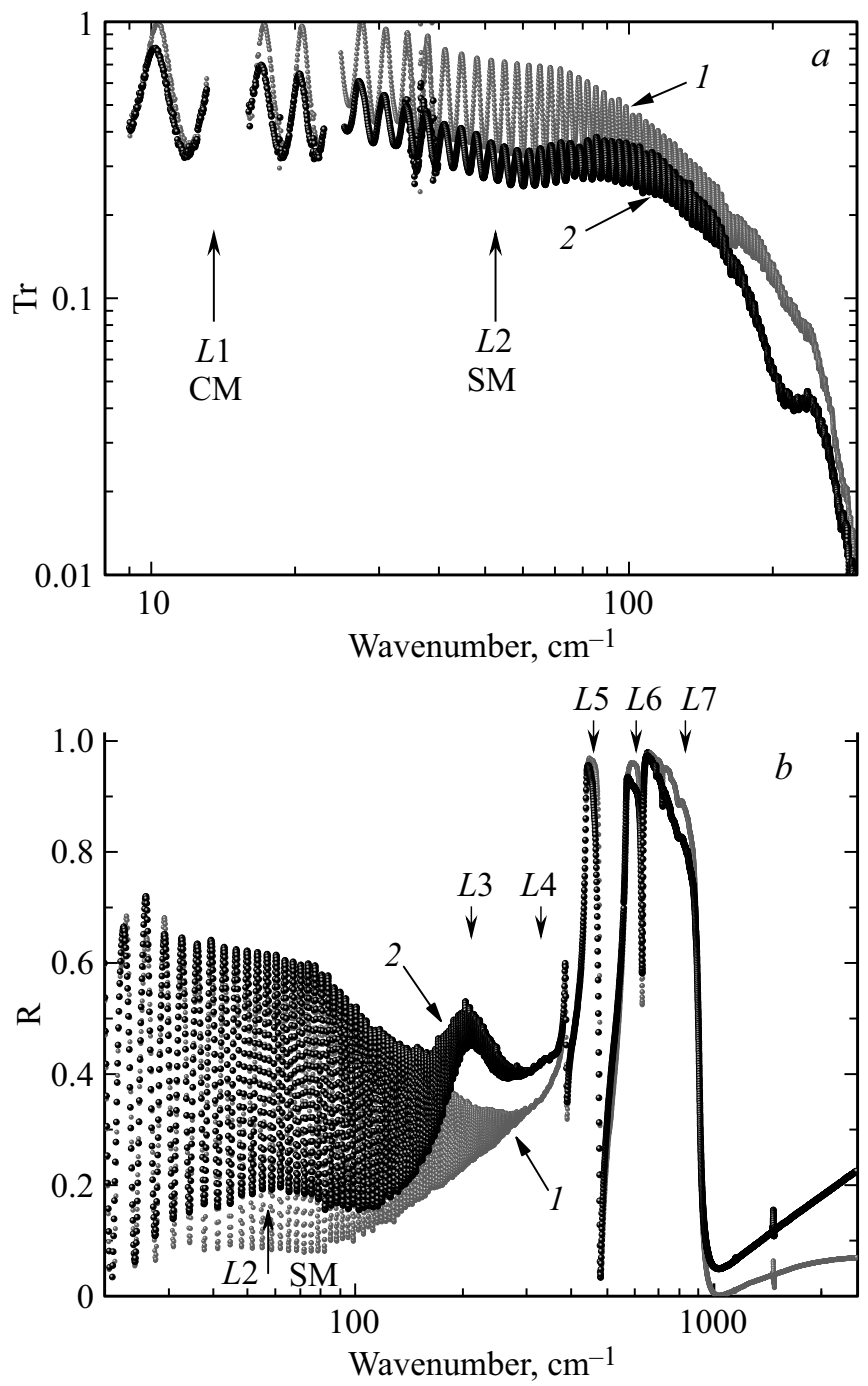

Рис. 6. Спектры пропускания $(a)$ и отражения $(b)$ подложки $\alpha-\mathrm{Al}_{2} \mathrm{O}_{3}$ (линия 1) и закристаллизованной пленки PZT на сапфировой подложке (линия 2). Толщина пленки $\sim 250 \mathrm{~nm}$. Стрелками обозначены полосы поглощения в пленке. СМ центральная мода, SM - мягкая мода.
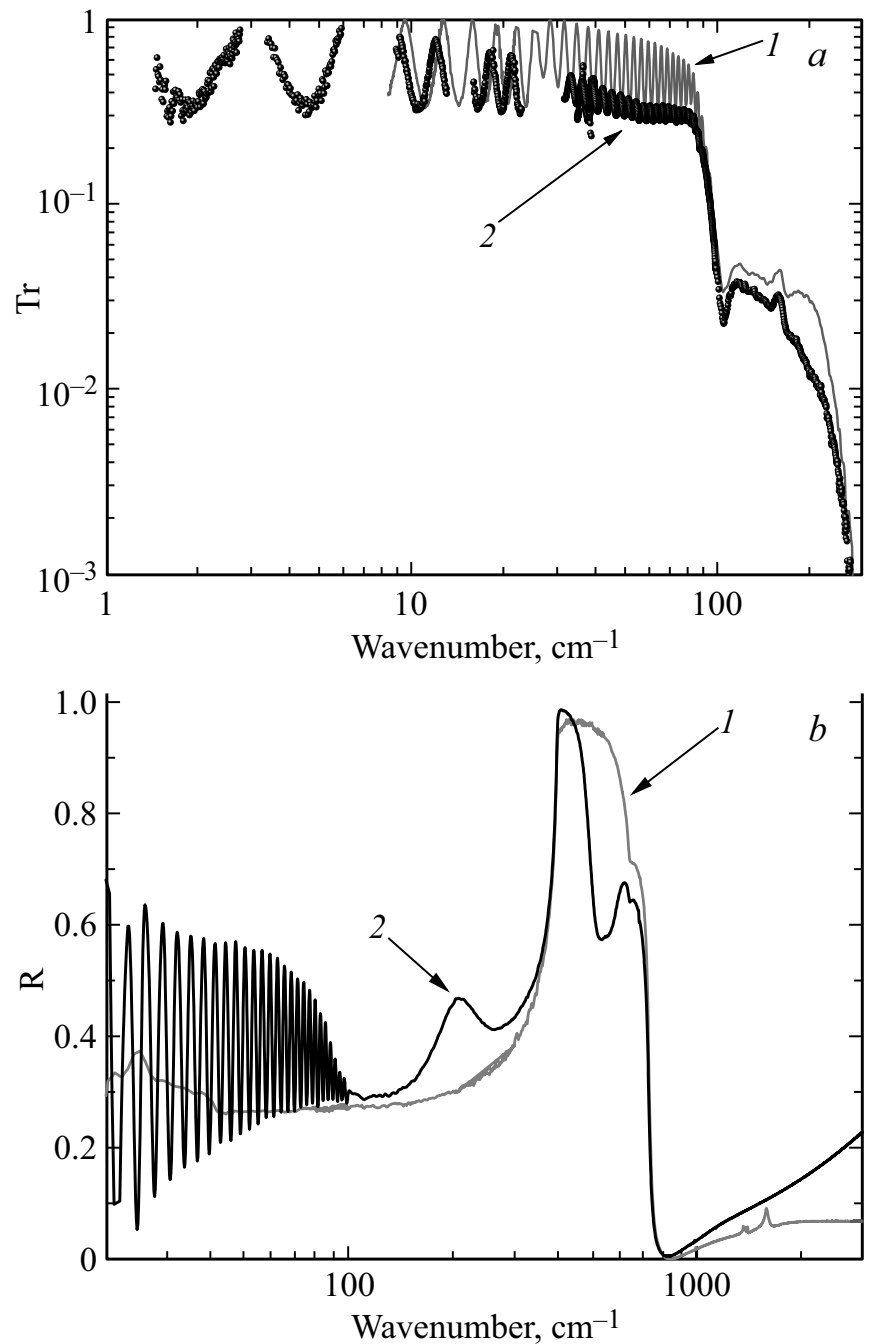

Pис. 7. Спектры пропускания $(a)$ и отражения $(b)$ подложки $\mathrm{MgO}(1)$ и пленки PZT на подложке $\mathrm{MgO}(2)$. Толщина пленки $\sim 250 \mathrm{~nm}$.

кой моды. Отметим, что на точность определения этих параметров также влияет и наличие в спектре сапфира многофононного разностного поглощения [42] на тех же самых частотах, где наблюдается дисперсия, обусловленная мягкой модой в пленках PZT.

На рис. 7 для сравнения показаны аналогичные спектры пропускания и отражения для подложки из оксида магния и подложки из оксида магния с нанесенной на нее закристаллизованной пленкой PZT.

На рис. 8 представлены спектр отражения закристаллизованной пленки PZT на подслое платины, измеренный в $p$-поляризации, и спектр, рассчитанный по модели классического гармонического осциллятора. Интерференция в высокочастотной части спектра определена оптическими и геометрическими параметрами пленки:

$$
2 d \sqrt{n^{2}-\sin ^{2} \alpha}=m \lambda=1 / \Delta v,
$$

где $d-$ толщина пленки, $m-$ порядок интерференции, $\lambda$ - длина волны, $n-$ показатель преломления пленки, 


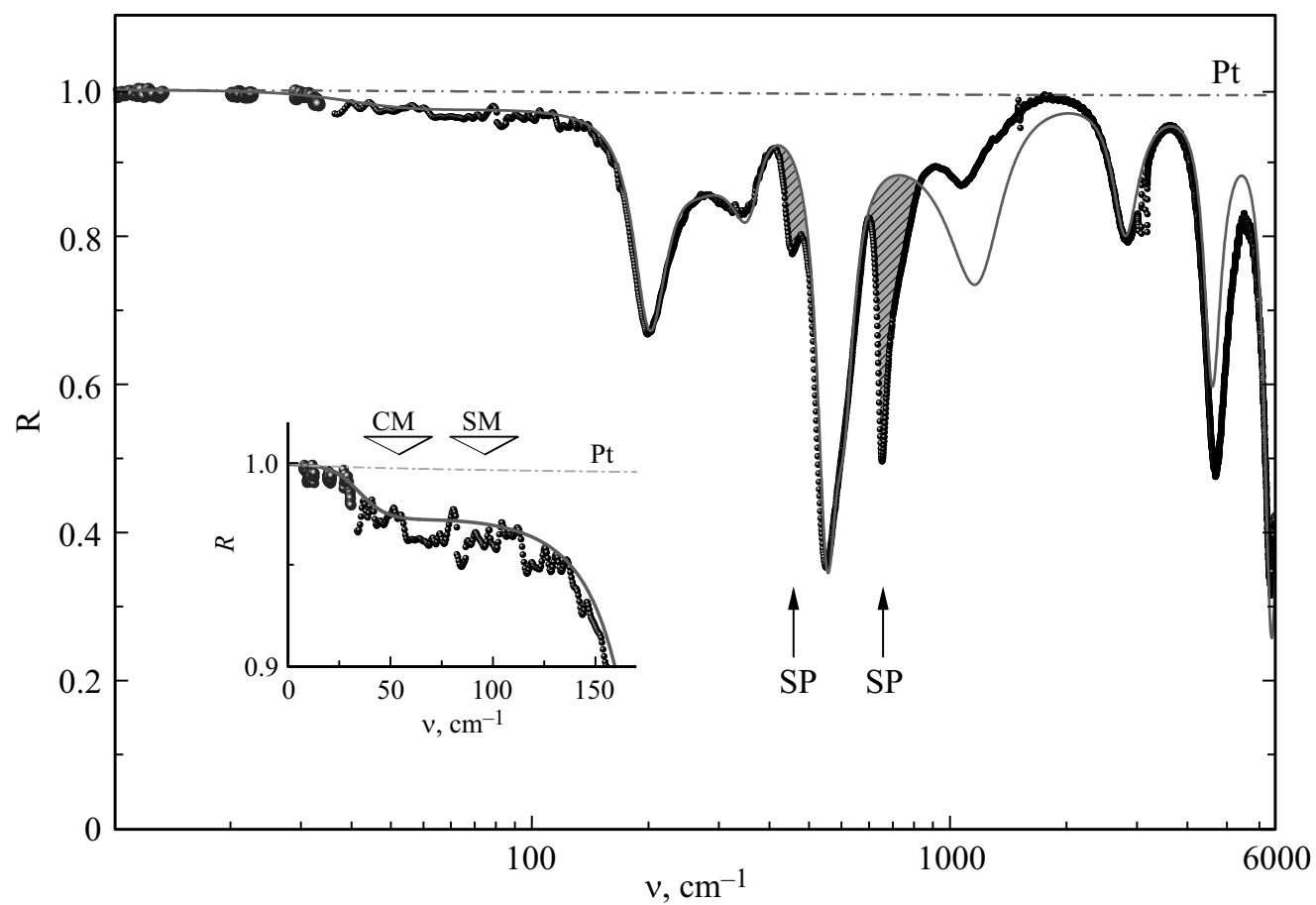

Рис. 8. Спектр отражения закристаллизованной пленки PZT на платине. Точки - экспериментальные данные для $p$-поляризации. Сплошная линия - расчет по модели классического осциллятора. Штрихпунктир - спектр отражения платины, рассчитанный по модели Друде. Стрелками SP - отмечены полосы поглощения, обусловленные поверхностными поляритонами. На вставке показана область дисперсии центральной $(\mathrm{CM})$ и мягкой $(\mathrm{SM})$ мод.

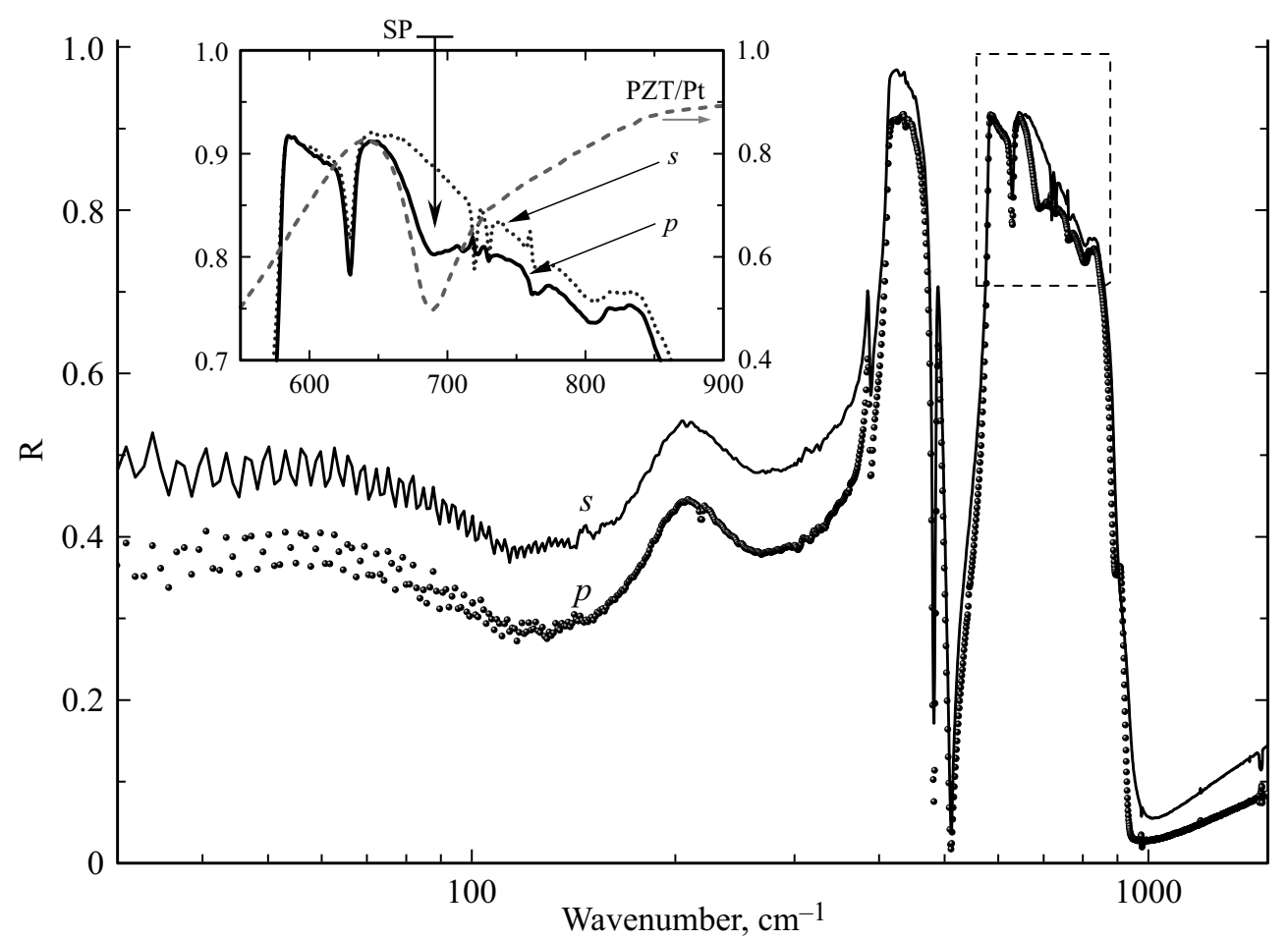

Рис. 9. Спектры отражения пленки PZT на подложке $\mathrm{Al}_{2} \mathrm{O}_{3}$ для $s$ - и p-поляризаций. На вставке показан участок частот, в котором $\varepsilon^{\prime}$ подложки отрицательна: пик поглощения поверхностного поляритона SP зарегистрирован в $p$-поляризации - сплошная линия; точками показан спектр в $s$-поляризации; для сравнения пунктиром показан спектр отражения пленки РZT на платине в p-поляризации. 
$\Delta v-$ расстояние между минимумами интерференции (в волновых числах $\mathrm{cm}^{-1}$ ), $\alpha-$ угол падения излучения.

Расхождение модельного спектра отражения с экспериментом на высокочастотном краю диапазона, выше $3000 \mathrm{~cm}^{-1}$, обусловлено дисперсией отражения слоя платины, не учтенной в расчетах, поскольку для определения высокочастотного диэлектрического вклада $\varepsilon_{\infty}$ использованы положения минимумов интерференции на частотной оси. Несоответствие модели и эксперимента в области $1000-2000 \mathrm{~cm}^{-1}$ проявляется в большем коэффициенте пропускания экспериментального спектра. Этот факт связан с ограничением модели классического осциллятора, в которой затухание лоренциана полагается константой. Как показано в работе [54], контур резонанса асимметричен, и на высоких частотах его вклад в поглощение убывает быстрее, чем в случае лоренцевого контура.

Приведенный на рис. 8 спектр получен для $p$-поляризованного излучения при угле падения $\approx 30$ градусов. Для диапазона 2000-4000 $\mathrm{cm}^{-1}$ усредненное значение показателя преломления можно оценить из интерференции: $n \approx 2.58 \pm 0.05$. Параметры полос поглощения в диапазоне $100-1000 \mathrm{~cm}^{-1}$ рассчитаны с хорошей точностью по модели классического осциллятора. В $\mathrm{THz}$ области ниже $100 \mathrm{~cm}^{-1}$ слабая и широкая полосы в спектре отражения соответствуют полосе, смоделированной двумя осцилляторами с большим диэлектрическим вкладом. В спектре присутствуют две линии (SP), для которых нет соответствующих фононов в спектре керамики, и они отсутствуют в спектрах, измеренных в $s$-поляризации. Эти полосы соответствуют поверхностным (интерфейсным) поляритонам на границе раздела пленка PZT/платина. Условием формирования в спектре полосы поверхностного поляритона является отрицательное значение действительной части диэлектрической проницаемости подложки [45]. В случае металлического подслоя платины это условие в IR-диапазоне выполняется всегда. Для диэлектрических подложек также возможна реализация условий для формирования интерфейсного поляритона.

Для проверки данного предположения мы провели измерения пленки PZT, осажденной на подложку из сапфира. Результаты измерений показаны на рис. 9. Область остаточных лучей соответствует расщеплению TO-LO фононов $570-900 \mathrm{~cm}^{-1}$, что по соотношению Лиддена-Сакса-Теллера дает диэлектрический вклад $\Delta \varepsilon \approx 3$. В этом диапазоне частот действительная часть диэлектрической проницаемости $\varepsilon^{\prime}(v)$ отрицательна и, таким образом, удовлетворяется условие для возбуждения интерфейсных поляритонов. Выделение пика поглощения проведено по стандартной схеме измерения коэффициента отражения в $s$ - и $p$-поляризованном излучении при угле падения 30 градусов.

Влияние степени пористости пленки на функцию диэлектрического отклика заключается в последовательном уменьшении эффективной диэлектрической проницаемости (рис. 10) [55]. Отсутствие данных для осцилля-

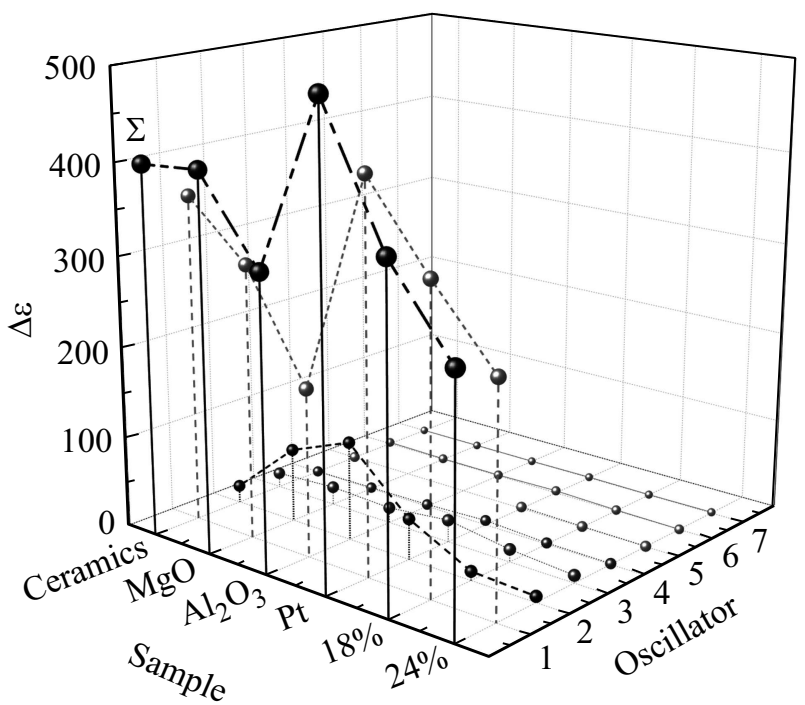

Рис. 10. Зависимости диэлектрических вкладов осцилляторов от типа образца. Данные для керамического образца рассчитаны по данным работы [19]. Суммарный вклад всех IR-активных мод $\Sigma=\Sigma \Delta \varepsilon_{j}+\varepsilon_{\infty}$ в зависимости от образца показан в положении „нулевого“ осциллятора.

тора $\mathrm{N} 5$ пленки на подложках $\mathrm{MgO}$ и $\mathrm{Al}_{2} \mathrm{O}_{3}$ объясняется тем, что частоты этого осциллятора в пленке близки к частотам фононов в этих подложках. Из-за большой ширины резонансных линий при комнатной температуре рассчитать параметры этого осциллятора с разумной точностью не представляется возможным.

Полярные характеристики пленок отражают зависимость диэлектрических вкладов осцилляторов от типа подложки и пленки. Плотные пленки PZT на сапфире и оксиде магния имеют те же величины вкладов, что и керамический образец. Расхождения могут быть обусловлены точностью определения параметров $\sim 10 \%$ и влиянием гетероэпитаксиальных напряжений. Центральная и мягкая мода для всех образцов дают определяющий вклад в низкочастотную проницаемость. Существенно большее значение проницаемости зарегистрировано для пленки на слое платины. Определение параметров низкочастотных возбуждений для такой конфигурации образца возможно с худшей, чем в остальных случаях точностью. Тем не менее, даже в пределах увеличенной погрешности различия этих вкладов регистрируются уверенно.

На рис. 11 представлены изменения собственных частот фононов в зависимости от исследуемого образца гетероструктуры. Учитывая тот факт, что рассогласования параметров ячейки различных подложек с пленкой значительны, есть все основания считать существенным влияние интерфейсных эффектов на колебательный спектр пленки. Необходимо отметить, что максимальные различия в величинах частот зарегистрированы в плотной пленке на платине. Для остальных образцов собственные частоты осцилляторов различа- 


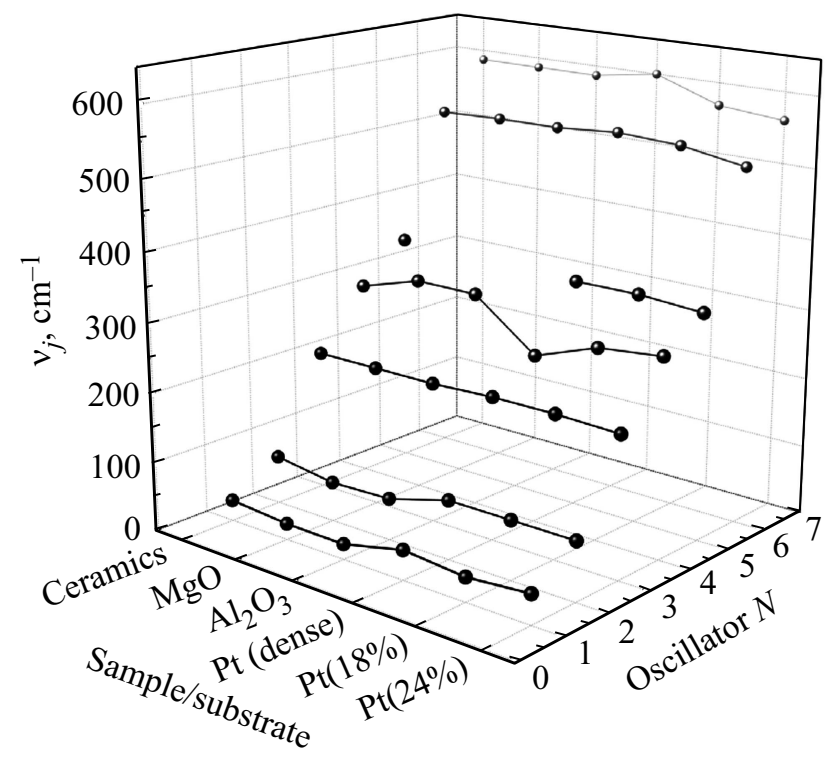

Рис. 11. Зависимости частот модельных осцилляторов для плотных пленок PZT на подложках $\mathrm{Al}_{2} \mathrm{O}_{3}, \mathrm{MgO}$ и пленок с различной степенью пористости на Pt. Для сравнения приведены данные из работы [19] для керамики эквивалентного состава.

ются менее выраженно. Сильнее всего это проявляется для осциллятора N4 в области $300 \mathrm{~cm}^{-1}$. В спектрах пропускания всех образцов полоса на этих частотах регистрируется надежно, что минимизирует погрешности определения параметров модельных осцилляторов. Расхождение частот для пленки на платине неожиданно, поскольку рассогласование параметров кристаллических ячеек платины и пленки минимальны, и можно было бы ожидать минимального расхождения с величинами частот керамического образца. Интерпретация этого явления, на наш взгляд, возможна на основании анализа собственных векторов полярных колебаний с учетом их взаимодействия с проводящим подслоем платины.

\section{Заключение}

Выяснены особенности диэлектрического отклика пленок PZT в составе различных гетероструктур в $\mathrm{THz}-\mathrm{IR}$-диапазоне, определяемые изменениями в параметрах IR-активных полос поглощения в зависимости от типа подложки и степени пористости пленки. Определено, что плотные пленки PZT с концентрацией $\mathrm{Ti}-\mathrm{Zr}$ (0.48-0.52), соответствующей максимальным величинам низкочастотной диэлектрической проницаемости, имеют качественное сходство функции диэлектрического отклика, как между собой, так и с керамическими образцами эквивалентного состава. Установлено, что для исследованных пленок на всех типах подложек формируются высокие значения диэлектрической проницаемости, которая меняется в пределах $\sim 25 \%$ относительно проницаемости керамического образца. Различия диэлектрической проницаемости и параметров электродипольных возбуждений в этих пленках представляют фундаментальный интерес при изучении влияния гетероэпитаксиальных напряжений на фононный спектр.

\section{Список литературы}

[1] U. Böttger, G. Arlt. Ferroelectrics 127, 95 (1992).

[2] B. Jaffe, W.R. Cook, H. Jaffe. // In: Piezoelectric Ceramics. Academic Press, London (1971). P. 328.

[3] B. Noheda, D.E. Cox, G. Shirane, R. Guo, B. Jones, L.E. Cross. Phys. Rev. B 63, 014103 (2000).

[4] D.I. Woodward, J. Knudsen, I.M. Reaney. Phys. Rev. B 72, 104110 (2005).

[5] J.F. Scott. Science 315, 954 (2007).

[6] K.A. Vorotilov, A.S. Sigov. Phys. Solid State 54, 894 (2012).

[7] S. Trolier-McKinstry, P. Muralt. J. Electroceramics 12, 7 (2004).

[8] C.B. Eom, S. Trolier-McKinstry. MRS Bulletin 37, 1007 (2012).

[9] J.B. Chang, H. Miyazoe, M. Copel, P.M. Solomon, X.-H. Liu, T.M. Shaw. A.G. Schrott, L.M. Gignac, G.J. Martyna, D.M. Newns. Nanotechnology 26, 375201 (2015).

[10] R.W. Whatmore. J. Electroceram. 13, 139 (2004).

[11] J.C. Park, S. Khym, J.Y. Park. Appl. Phys. Lett. 102, 043901 (2013).

[12] J.-M. Hu, L.-Q. Chen, C.-W. Nan. Adv. Mater. 28, 15 (2016).

[13] N. Tiercelin, Y. Dusch, V. Preobrazhensky, P. Pernod. J. Appl. Phys. 109, 07D726 (2011).

[14] N. Lei, T. Devolder, G. Agnus, P. Aubert, L. Daniel, J.-V. Kim, W. Zhao, T. Trypiotis, R.P. Cowburn, C. Chappert, D. Ravelosona, P. Lecoeur. Nature Commun. 4, 1378 (2013).

[15] I. Tomeno, Y. Ishii, Y. Tsunoda, K. Oka. Phys. Rev. B 73, 064116 (2006).

[16] I. Tomeno, J.A. Fernandes-Baca, K.J. Marty, Y. Tsinoda, K. Oka. J. Phys: Conf. Ser. 340, 012054 (2012).

[17] J. Hlinka, M. Kempa, J. Kulda, P. Bourges, A. Kania, J. Petzelt. Phys. Rev. B 73, 140101(R) (2006).

[18] A.G. Souza Filho, K.C.V. Lima, A.P. Ayala, I. Guedes, P.T.C. Freire, F.E.A. Melo, J. Mendes Filho, E.B. Araújo, J.A. Eiras. Phys. Rev. B 66, 132107 (2002).

[19] E. Buixaderas, I. Gregora, M. Savinov, J. Hlinka, L. Jin, D. Damjanovic, B. Malic. Phys. Rev. B 91, 014104 (2015).

[20] A.K. Tagantsev, K. Vaideeswaran, S.B. Vakhrushev, A.V. Filimonov, R.G. Burkovsky, A. Shaganov, D. Andronikova, A.I. Rudskoy, A.Q.R. Baron, H. Uchiyama, D. Chernyshov, A. Bosak, Z. Ujma, K. Roleder, A. Majchrowski, J.-H. Ko, N. Setter. Nature Commun. 4, 2229 (2013).

[21] R.G. Burkovsky, A.K. Tagantsev, K. Vaideeswaran, N. Setter, S.B. Vakhrushev, A.V. Filimonov, A. Shaganov, D. Andronikova, A.I. Rudskoy, A.Q.R. Baron, H. Uchiyama, D. Chernyshov, Z. Ujma, K. Roleder, A. Majchrowski, J.-H. Ko. Phys. Rev. B 90, 144301 (2014).

[22] J. Hlinka, T. Ostapchuk, E. Buixaderas, C. Kadlec, P. Kuzel, I. Gregora, J. Kroupa, M. Savinov, A. Klic, J. Drahokoupil, I. Etxebarria, J. Dec. Phys. Rev. Lett. 112, 197601 (2014).

[23] E. Buixaderas, D. Nuzhyy, J. Petzelt, Li Jin, D. Damjanovic. Phys. Rev. B 84, 184302 (2011). 
[24] I. Fedorov, J. Petzelt, V. Železný, G.A. Komandin, A.A. Volkov, K. Brooks, Y. Huang, N. Setter. J. Phys.: Condens. Matter 7, 4313 (1995).

[25] V. Železný, I. Fedorov, J. Petzelt. Czech. J. Phys. 48, 537 (1998).

[26] S. Gilbert, S. Hunter, D. Ritchey, C. Chi, D. Tayer, J. Amanao, S. Aggarwal, T. Moise, T. Sakoda, S. Summerfelt, K. Singh, C. Kazemi, D. Carl, B. Bierman. J. Appl. Phys. 93, 1713 (2003).

[27] H. Funakubo. Topics Appl. Phys. 93, 95 (2004).

[28] C. Yang, J. Liu, S. Zhang, Z. Chen. Mater. Sci. Eng. B 99, 356 (2003).

[29] I.P. Pronin, E.Ju. Kaptelov, S.V. Senkevich, V.A. Klimov, N.V. Zajceva, T.A. Shaplygina, V.P. Pronin, S.A. Kukushkin. Phys. Solid State 52, 124 (2010).

[30] M. Adachi. Topics Appl. Phys. 98, 91 (2005).

[31] V.M. Mukhortov, Y.I. Golovko, G.N. Tolmachev, A.N. Klevtzov. Ferroelectrics 247, 75 (2000).

[32] T. Morita, T. Kanda, Y. Yamagata, M. Kurosawa, T. Higuchi. Jpn. J. Appl. Phys. 36, 2998 (1997).

[33] Th. Schneller, R. Waser, M. Kosec, D. Payne. Chemical Solution Deposition of Functional Oxide Thin Films. Springer (2013).

[34] K.A. Vorotilov, M.I. Yanovskaya, E.P. Turevskaya, A.S. Sigov. J. Sol-Gel Sci. Technology 16, 109 (1999).

[35] X. Gu, N. Izyumskaya, V. Avrutin, H. Morko, T.D. Kang, H. Lee. Appl. Phys. Lett. 89, 122912 (2006).

[36] J. Petzelt, S. Kamba. Mater. Chem. Phys. 79, 175 (2003).

[37] G.A. Komandin, O.E. Porodinkov, I.E. Spektor, L.D. Iskhakova, A.A. Bush, K.E. Kamentsev, D.S. Seregin. Phys. Solid State, 59, 1094 (2017).

[38] G.A. Komandin, O.E. Porodinkov, A.A. Bush, A.F. Koroleva, B.E. Spektor, S.V. Chuchupal, D.S. Seregin, K.D. Iskhakova. ФTT 59, 2343 (2017).

[39] J. Petzelt, S. Kamba. Ferroelectrics 503, 19 (2016).

[40] G.V. Kozlov, A.A. Volkov. Topics Appl. Phys. 74, 51 (1998).

[41] G.A. Komandin, O.E. Porodinkov, I.E. Spektor, A.A. Volkov. Phys. Solid State 51, 10, 2045 (2009).

[42] G.A. Komandin, O.E. Porodinkov, L.D. Iskhakova, I.E. Spektor, A.A. Volkov, K.A. Vorotilov, D.S. Seregin, A.S. Sigov. Phys. Solid State 56, 11, 2206 (2014).

[43] G.A. Komandin, A.A. Volkov, I.E. Spektor, K.A. Vorotilov, V.M. Mukhortov. Phys. Solid State 51, 7, 1351 (2009).

[44] Handbook of optical constant of Solids / Ed. E.D. Palik. Academic Press, San Diego (1985). V. 1. P. 804.

[45] G.N. Zhizhin, M.A. Moskalova, E.V. Shomina, V.A. Yakovlev. Surface Electromagnetic wave on metal surfaces. In Surface polaritons. Electromagnetic wave at surfaces and Interfaces / Eds V.M. Agranovich, D.L. Mills. Amsterdam, N.Y., Oxford. North-Holland Publishing Company (1982). P. 93-144.

[46] K. Vorotilov, A. Sigov, D. Seregin, Yu. Podgorny, O. Zhigalina, D. Khmelenin. Phase Transitions 86, 11, 1152 (2013).

[47] T. Ostapchuk, J. Petzelt, V. Železný, A. Pashkin, J. Pokorný, I. Drbohlav, R. Kužel, D. Rafaja, B.P. Gorshunov, M. Dressel, Ch. Ohly, S. Hoffmann-Eifert, R. Waser. Phys. Rev. B 66, 235406 (2002).

[48] G.A. Komandin, V.M. Mukhortov, O.E. Porodinkov, I.E. Spektor. Phys. Solid State, 55, 2, 288 (2013).

[49] В.М. Мухортов, Ю.И. Головко, Ю.И. Юзюк. УФН 179, 8 , 909 (2009).
[50] N. Izumskaya, Y.-I. Alivov, S.-J. Cho, H. Morkoc, H. Lee, Y.S. Kang. Crit. Rev. Solid State Mater. Sci. 32, 3-4, 111 (2007).

[51] D. Nuzhnyy, J. Petzelt, S. Kamba, P. Kužel, C. Kadlec, V. Bovtun, M. Kempa, J. Schubert, C.M. Brooks, D.G. Schlom. Appl. Phys. Lett. 95, 232902 (2009).

[52] J.H. Haeni, P. Irvin, W. Chang, R. Uecker, P. Reiche, J.L. Li, S. Choudhury, W. Tian, M.E. Hawley, B. Craigo, A.K. Tagantsev, X.Q. Pan, S.K. Streiffer, L.Q. Chen, S.W. Kirchoefer, J. Levi, D.G. Schlom. Nature 430, 758 (2004).

[53] V. Goian, S. Kamba, N. Orloff, T. Birol, C.H. Lee, D. Nuzhnyy, J.C. Booth, M. Bernhagen, R. Uecker, D.G. Schlom. Phys. Rev. B 90, 174105 (2014).

[54] H. Bilz, L. Genzel, H. Happ. Z. Phys. 160, 535 (1960).

[55] G.A. Komandin, O.E. Porodinkov, I.E. Spektor, A.A. Volkov, K.A. Vorotilov, D.S. Seregin, A.S. Sigov. Phys. Status Solidi C 14, 1-2, 1600211 (2017). 